\title{
Effects of two consecutive mixed meals high in palmitic acid or stearic acid on 8-h postprandial lipemia and glycemia in healthy-weight and overweight men and postmenopausal women: a randomized controlled trial
}

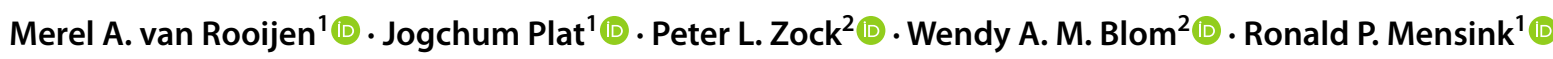

Received: 26 November 2020 / Accepted: 26 February 2021 / Published online: 17 March 2021

(c) The Author(s) 2021

\begin{abstract}
Purpose Palmitic and stearic acids have different effects on fasting serum lipoproteins. However, the effects on postprandial lipemia and glycemia are less clear. Also, the effects of a second meal may differ from those of the first meal. Therefore, we studied the effects of two consecutive mixed meals high in palmitic acid- or stearic acid-rich fat blends on postprandial lipemia and glycemia.

Methods In a randomized, crossover study, 32 participants followed 4-week diets rich in palmitic or stearic acids, At the end of each dietary period, participants consumed two consecutive meals each containing $\pm 50 \mathrm{~g}$ of the corresponding fat blend. Results Postprandial concentrations of triacylglycerol (diet-effect: $-0.18 \mathrm{mmol} / \mathrm{L} ; p=0.001$ ) and apolipoprotein B48 (dieteffect: $-0.68 \mathrm{mg} / \mathrm{L} ; p=0.002$ ) were lower after stearic-acid than after palmitic-acid intake. Consequently, total (iAUC ${ }_{0-8 \mathrm{~h}}$ ) and first meal $\left(\mathrm{iAUC}_{0-4 \mathrm{~h}}\right)$ responses were lower after stearic-acid intake $(p \leq 0.01)$. Second meal responses ( $\left.\mathrm{iAUC}_{4-8 \mathrm{~h}}\right)$ were not different. Postprandial changes between the diets in non-esterified fatty acids (NEFA) and C-peptide differed significantly over time ( $p<0.001$ and $p=0.020$ for diet $*$ time effects, respectively), while those for glucose and insulin did not. The dAUC $0-8 \mathrm{~h}, \mathrm{dAUC}_{0-4} \mathrm{~h}$, and dAUC $\mathrm{AL}_{4-8 \mathrm{~h}}$ for NEFA were larger after stearic-acid intake $(p \leq 0.05)$. No differences were observed in the iAUCs of C-peptide, glucose, and insulin. However, second meal responses for glucose and insulin (iAUC i-8 $_{\text {h) }}$ tended to be lower after stearic-acid intake $(p<0.10)$.

Conclusion Consumption of the stearic acid-rich meals lowered postprandial lipemia as compared with palmitic acid. After the second stearic acid-rich meal, concentrations of C-peptide peaked earlier and those of NEFA decreased more.

Clinical trial registry This trial was registered at clinicaltrials.gov as NCT02835651 on July 18, 2016.
\end{abstract}

Keywords Stearic acid $\cdot$ Palmitic acid $\cdot$ Human intervention study $\cdot$ Postprandial $\cdot$ Lipemia $\cdot$ Glycemia

\section{Introduction}

As we spend most of the day in a postprandial state, understanding relations between diet-induced postprandial physiological changes and cardiometabolic health is important.

Ronald P. Mensink

r.mensink@maastrichtuniversity.nl

1 Department of Nutrition and Movement Sciences, NUTRIM (School of Nutrition and Translational Research in Metabolism), Maastricht University Medical Center, Maastricht, The Netherlands

2 Unilever Research and Development, Wageningen, The Netherlands
Indeed, it has been shown that elevated and prolonged postprandial lipemia and glycemia are associated with an increased risk to develop cardiovascular disease (CVD) [1, 2]. However, for dietary fat intake, recommendations are mainly based on effects on fasting serum LDL-cholesterol (LDL-C) concentrations, an established CVD-risk factor [3]. It is for example well-known that replacing saturated fatty acids with unsaturated fatty acids has a beneficial effect on LDL-C [4]. Saturated fat, however, is an umbrella term for different saturated fatty acids that exert different metabolic effects. Of these, palmitic acid (C16:0) and stearic acid (C18:0) are the most commonly consumed saturated fatty acids in the Western diet. It is well established that palmitic acid increases fasting serum LDL-C 
concentrations compared with stearic acid. However, the effects of these saturated fatty acids on postprandial metabolism are less clear. Attenuated postprandial lipemia after acute intake of stearic acid compared with palmitic acid has been observed in two studies [5, 6], but not in other studies [7-11]. One hypothesis is that stearic acidrich fats delay fat digestion and absorption, because of the presence of more fat solids at body temperature due to its higher melting range [12]. So far, no differences between palmitic-acid and stearic-acid intake have been found in postprandial responses of glucose $[6,13]$ and insulin $[5$, $8,13]$.

In daily-life, people generally consume multiple meals a day and lipids ingested during the first meal will also appear in the circulation when a second meal is consumed, even if this second meal is low in fat $[14,15]$. Previous studies examining acute effects of palmitic-acid versus stearicacid intakes provided a low-fat lunch after 3 to $4 \mathrm{~h}$ [6-9]. However, as most meals during the day provide fats, it is of interest to examine if postprandial responses of consecutive meals high in fat differ from those after a single meal. Therefore, we have examined the effects of two consecutive mixed meals high in palmitic acid- or stearic acid-rich fat blends on postprandial lipemia and glycemia during an 8-h period. Postprandial tests were performed after subjects had consumed 4-week diets rich in the corresponding fatty acid.

\section{Methods}

This postprandial intervention study was part of a doubleblind, randomized, crossover study that consisted of two 4-week intervention periods during which healthy-weight or overweight men and women received products enriched with either palmitic acids (C16:0) or stearic acids (C18:0). Effects of the 4-week diets on fasting cardiometabolic risk markers and details of the study design have been described previously [16]. Compared with the diet rich in palmitic acid, intake of palmitic acid was 6.0 percent of energy $(\mathrm{En} \%)$ lower when subjects consumed the diet rich in the stearic acid diet $(p<0.001)$ and intake of stearic acid 6.5 En\% higher. Oleic-acid intake was slightly higher on the stearic-acid diet (+0.4 En\%) and fiber intake was - $1.3 \mathrm{~g}$ lower. Intakes of energy, other macronutrients, and cholesterol were comparable between the two intervention periods, which were separated by a wash-out period of at least 4 weeks (Supplemental table 1). At the end of each intervention period, an 8 -h postprandial test was performed for which participants consumed a mixed meal high in either palmitic acid- or stearic acid-rich fat blends. Participants received a second meal $4 \mathrm{~h}$ after the first meal to induce a second-meal effect.

\section{Participants}

Briefly, healthy men and women were recruited from Maastricht and surrounding areas and met the following criteria: aged between 45 and 70 years, postmenopausal (women), BMI between 18 and $30 \mathrm{~kg} / \mathrm{m}^{2}$ with a stable body weight during the last 3 months $(<3 \mathrm{~kg}$ change), no cardiovascular disease or medical condition that might interfere with the study. Participants were included if they were healthy, which was based on a medical questionnaire, had fasted serum total cholesterol (TC) concentrations $<8.0 \mathrm{mmol} / \mathrm{L}$ and triacylglycerol (TAG) concentrations $<4.5 \mathrm{mmol} / \mathrm{L}$, and plasma $\mathrm{HbA} 1 \mathrm{c}$ concentrations $<48 \mathrm{mmol} / \mathrm{mol}$ (or $6.5 \%$ ). After screening, 41 participants were included. All participants gave their written informed consent before entering the study. The Medical Ethical Committee of the MUMC + had approved the protocol. The study was registered at ClinicalTrials.gov with identifier NCT02835651.

\section{Study design and meals}

For the postprandial test at the end of each intervention period, participants were asked to refrain from strenuous exercise $48 \mathrm{~h}$ before this test day. After measuring weight, blood pressure, and obtaining a fasted blood sample via venepuncture, an intravenous cannula was placed in an antecubital vein and another fasting blood sample was collected (T0). Participants then received a mixed meal provided as a shake, which they were asked to consume within $5 \mathrm{~min}$. This meal contained $46.6 \mathrm{~g}$ of the fat blend that participants also received during the preceding 4-week intervention period. The composition of the meal was similar for all participants and each meal consisted of $50 \mathrm{~g}$ of fat, $5 \mathrm{~g}$ of protein, and $54 \mathrm{~g}$ of carbohydrates (Table 1). This amount of fat was chosen, because it represents a realistic fat load in a Western dinner and causes a clear increase in serum TAG concentrations [17]. $4 \mathrm{~h}$ after the first meal, participants consumed a second meal with the same composition as the first one. Postprandial blood samples were taken at 15 (T15), 30 (T30), 45 (T45), 60 (T60), 90 (T90), 120 (T120), 180 (T180), 240 (T240), 300 (T300), 360 (T360), 420 (T420), and 480 (T480) minutes after shake consumption. Immediately after T240 (approximately around lunch time), the second meal was consumed. During the entire test day, participants were not allowed to drink anything-except for water-or to eat. Participants were asked to keep the amount of water consumption comparable between both test days. 
Table 1 Nutrient composition of the standardized test shakes provided for breakfast and lunch

\begin{tabular}{lll}
\hline & $\begin{array}{l}\text { Palmitic acid- } \\
\text { rich shake }\end{array}$ & Stearic acid-rich shake \\
\hline Energy (kcal) & 697.4 & 697.4 \\
Carbohydrates (en\%) & 30.8 & 30.8 \\
Protein (en\%) & 4.8 & 4.8 \\
Fat (en\%) & $64.5(50 \mathrm{~g})$ & $64.5(50 \mathrm{~g})$ \\
SFA (en\%) & 33.0 & 33.0 \\
C16:0 (en\%) & $28.3(22 \mathrm{~g})$ & $3.1(2.5 \mathrm{~g})$ \\
C18:0 (en\%) & $3.2(2.4 \mathrm{~g})$ & $29.2(22.6 \mathrm{~g})$ \\
MUFA (en\%) & 26.0 & 26.4 \\
C18:1 (en\%) & 25.7 & 26.1 \\
PUFA (en\%) & 4.7 & 4.7 \\
C18:3 n-3 (en\%) & 0.1 & 0.4 \\
Cholesterol (mg) & 120 & 120 \\
Fiber (g) & 1.28 & 1.28 \\
\hline
\end{tabular}

$S F A$, saturated fatty acids; $M U F A$, cis-monounsaturated fatty acids; $P U F A$, cis-polyunsaturated fatty acids

\section{Experimental fat blends}

Both blends of natural fats were provided by Unilever R\&D (Vlaardingen, Netherlands). For the palmitic acidrich blend, a mix of $90 \%$ palm oil mid-fraction (POM) and $10 \%$ high oleic sunflower oil (HOSO) was used. For the stearic acid-rich blend, a mix of $92 \%$ allanblackia oil $(\mathrm{AB})$ and $8 \%$ sunflower oil (SO) was used. Fat blends were comparable in saturated, monounsaturated and polyunsaturated fatty acid content (Supplemental table 2). Slip melting points for the palmitic acid- and stearic acid-rich fat blend were respectively $33.9{ }^{\circ} \mathrm{C}$ and $40.5^{\circ} \mathrm{C}$, and the solid fat contents at $37^{\circ} \mathrm{C}$ were 1 and $8 \%$.

\section{Blood collection and biochemical analyses}

Blood was sampled in serum separator vacutainer tubes (Becton, Dickinson and company, NJ, USA) for analyses of triacylglycerol (TAG), apolipoprotein B48 (apoB48), non-esterified fatty acids (NEFA), insulin, and C-peptide. After sampling, serum tubes were allowed to clot for at least $30 \mathrm{~min}$ at room temperature and subsequently centrifuged at $1300 \times g$ for $15 \mathrm{~min}$ at $20^{\circ} \mathrm{C}$. Blood for glucose analysis was sampled in NaF-plasma vacutainer tubes (Becton, Dickinson and company) and directly put on ice after sampling with subsequent centrifugation at $1300 \times g$ for $15 \mathrm{~min}$ at $4{ }^{\circ} \mathrm{C}$. Aliquots of serum and plasma samples were snap-frozen in liquid nitrogen and stored at $-80{ }^{\circ} \mathrm{C}$ until analysis.

Serum concentrations of TAG corrected for free glycerol (GPO Trinder; Sigma-Aldrich, Missouri, USA) and apoB48 (ELISA; Shibayagi Co., Shibukawa Japan) were measured at all timepoints except for T15 and T45. Concentrations of serum NEFA (Wako Chemicals GmbH, Neuss, Germany), serum insulin and C-peptide (Linco Research, Missouri, USA), and plasma glucose (Horiba ABX) were measured at all time points. Samples from one subject were analyzed within the same analytical run.

\section{Statistical analyses}

The primary outcome parameter of this study was the change in fasting cholesterol efflux capacity for which a power calculation was performed (4). Here we report the results on other outcome parameters. Data are reported as least squared mean (LSM) with 95\% confidence interval (CI) unless otherwise indicated. Postprandial time curves were analyzed using linear mixed models with participants as between subject variable, baseline values of the corresponding day (T0) as the covariate, and period, diet, time, diet*time, and baseline as fixed factors. If the diet*time interaction term did not reach statistical significance, indicating that responses were similar at all time points, it was omitted from the model. In this model, the statistical significance of the factor diet indicated that differences between the stearic acid and palmitic acid diets were similar at all time points. Differences are reported as least squared means (LSM) with 95\% confidence interval (CI). Sex effects were also determined by addition of sex, diet*sex, time*sex, and diet*sex*time as fixed factors to the model. However, for none of the parameters sex effects were present and therefore omitted from the model. Incremental areas under the curve (iAUC) or decremental areas under the curve (dAUC) were calculated for all parameters using the trapezoidal rule as previously described [18]. We assessed the total postprandial response $(0$ to $8 \mathrm{~h}$; i/dAUC 0-8 $\mathrm{h}$ ), as well as the first meal response ( 0 to $4 \mathrm{~h}$; i/dAUC $0-4 \mathrm{~h}$ ) and the second meal response (4 to $8 \mathrm{~h}$; $\mathrm{i} / \mathrm{dAUC}_{4-8 \mathrm{~h}}$ ). Peak increases or decreases were calculated by comparing maximal changes during the $8 \mathrm{~h}$ postprandial follow-up to T0 $\left(\max _{0-8 \mathrm{~h}}\right)$. Maximal changes after the first meal were calculated by comparing concentrations between $\mathrm{T} 0$ and T240 to the concentrations at T0 $\left(\max _{0-4 \mathrm{~h}}\right)$ and maximal changes after the second meal were calculated by comparing concentrations between T240 and T480 to the concentrations at T240 $\left(\max _{4-8 \mathrm{~h}}\right)$. i/dAUC differences and maximal increases were assessed using linear mixed models with subject as a random factor, and period and diet as fixed factors, and reported as LSM with 95\% CI. Sex effects were also tested for i/dAUCs by addition of sex and diet*sex to the model as fixed factors but the diet*sex interaction term was not significant for any of the parameters and thus omitted. Results were considered statistically significant if $p<0.05$. All analyses were performed using IBM SPSS Statistics for Mac, version 24.0 (IBM Corp. Armonk, NY, USA). Blinding was maintained until all analyses were performed. 


\section{Results}

The participant flow throughout the study is shown in Supplemental fig. S1. Fifty-eight participants were assessed for eligibility of which 41 were included and randomly allocated to the intervention periods. Seven participants withdrew during the first week of the first intervention period as described previously [16]. Of the remaining 34 participants, one man and one woman did not complete one or both postprandial test day(s) due to nausea. In the end, 32 participants (19 men and 13 women) completed both postprandial test days and were included in the analyses. Of these, 10 men and 7 women started with the palmitic-acid diet, and 9 men and 6 women with the stearic-acid diet. Characteristics of the participants at screening are shown in Supplemental Table 3.

\section{Postprandial lipemia}

For postprandial TAG concentrations, there were no significant differences between the meals rich in palmitic acid or stearic acid at the various time points of the postprandial response ( $p=0.742$ for diet*time interaction; Fig. 1 ). However, a significant diet-effect was observed, i.e. postprandial TAG concentrations after stearic-acid intake were on average $0.18 \mathrm{mmol} / \mathrm{L}$ lower $(p=0.001)$ over the total 8 -h follow-up period. Consequently, the $\mathrm{iAUC}_{0-8 \mathrm{~h}}(p=0.002)$ and peak values $\left(\mathrm{TAG}_{\max 0-8 \mathrm{~h}} ; \mathrm{p}=0.003\right)$ were lower after stearic-acid intake (Supplemental table 4). During the $4 \mathrm{~h}$ after the first meal, comparable results were observed as over the whole 8-h follow-up period, i.e. the $\mathrm{iAUC}_{0-4 \mathrm{~h}}$ and TAG $\max 0-4 \mathrm{~h}$ were lower $(\mathrm{p}=0.007$ for both) after the stearic-acid meal. The $\mathrm{iAUC}_{4-8 \mathrm{~h}}$ in the last $4 \mathrm{~h}$ after the second meal was not statistically different between palmitic acid and stearic acid $(p=0.127)$, but peak values tended to be lower after stearic-acid intake $\left(\mathrm{TAG}_{\max 4-8 \mathrm{~h}} ; p=0.079\right)$.

Differences in postprandial changes at the various timepoints for apoB 48 were comparable $(p=0.451$ for diet*time interaction; Fig. 2). Like for TAG, average apoB48 concentrations were overall lower after stearic-acid than after palmitic-acid intake (diet-effect: $-0.68 \mathrm{mg} / \mathrm{L} ; p=0.002$ ), as also shown by the lower iAUC $\mathrm{A}_{0-8 \mathrm{~h}}(p=0.008)$ and peak values (ApoB $48_{\max 0-8 \mathrm{~h}} ; p=0.034$; Supplemental table 5). After the first meal, also a lower iAUC $\mathrm{AU}_{0-4 \mathrm{~h}}(p=0.010)$ and ApoB $48_{\max 0-4 \mathrm{~h}}(p=0.048)$ were observed after intake of stearic acid. After the second meal, the $\mathrm{iAUC}_{4-8 \mathrm{~h}}(\mathrm{p}=0.355)$ and ApoB $48_{\max 4-8 \mathrm{~h}}(p=0.585)$ were not different between palmitic-acid and stearic-acid intakes. Subsequently, postprandial changes in TAG:apoB48 ratio at the various timepoints were comparable between palmitic-acid and stearic-acid intakes $(p=0.237$ for diet*time interaction; Supplemental Fig. 2) and no diet-effects were observed $(p=0.881)$.

\section{Postprandial glycemia}

For postprandial glucose concentrations, postprandial responses after palmitic-acid and stearic-acid intakes at the various time points did not differ significantly ( $p=0.074$ for diet*time interaction; Fig. 3). Also, no diet-effects were observed $(p=0.503)$. The iAUC ${ }_{0-8 \mathrm{~h}}(p=0.375)$ and peak values (Glucose $\mathrm{max}_{\max 8 \mathrm{~h}} ; p=0.876$ ) were comparable between palmitic-acid and stearic-acid intakes (Supplemental table 6). After the first meal, the iAUC ${ }_{0-4 \mathrm{~h}}(p=0.362)$ was also not different, but the Glucose $\mathrm{max}_{\operatorname{ma}-4 \mathrm{~h}}$ tended to be higher after stearic-acid intake $(p=0.059)$. In contrast to
Fig. 1 Postprandial changes in triacylglycerols (TAG; $\mathrm{mmol} / \mathrm{L}$ ) over time after meals rich in palmitic acid $(\bullet)$ or stearic acid $(\diamond)^{\mathrm{a}}$. ${ }^{\mathrm{a}} \mathrm{TAG}$ concentrations were measured at baseline, and 30 , $60,90,120,180,240,300,360$, and $480 \mathrm{~min}$ after meal intake. After $240 \mathrm{~min}$, a second meal was consumed that was similar to the first meal. Postprandial time curves were analyzed using linear mixed models. $N=32$. A significant overall diet-effect was observed $(p=0.001)$

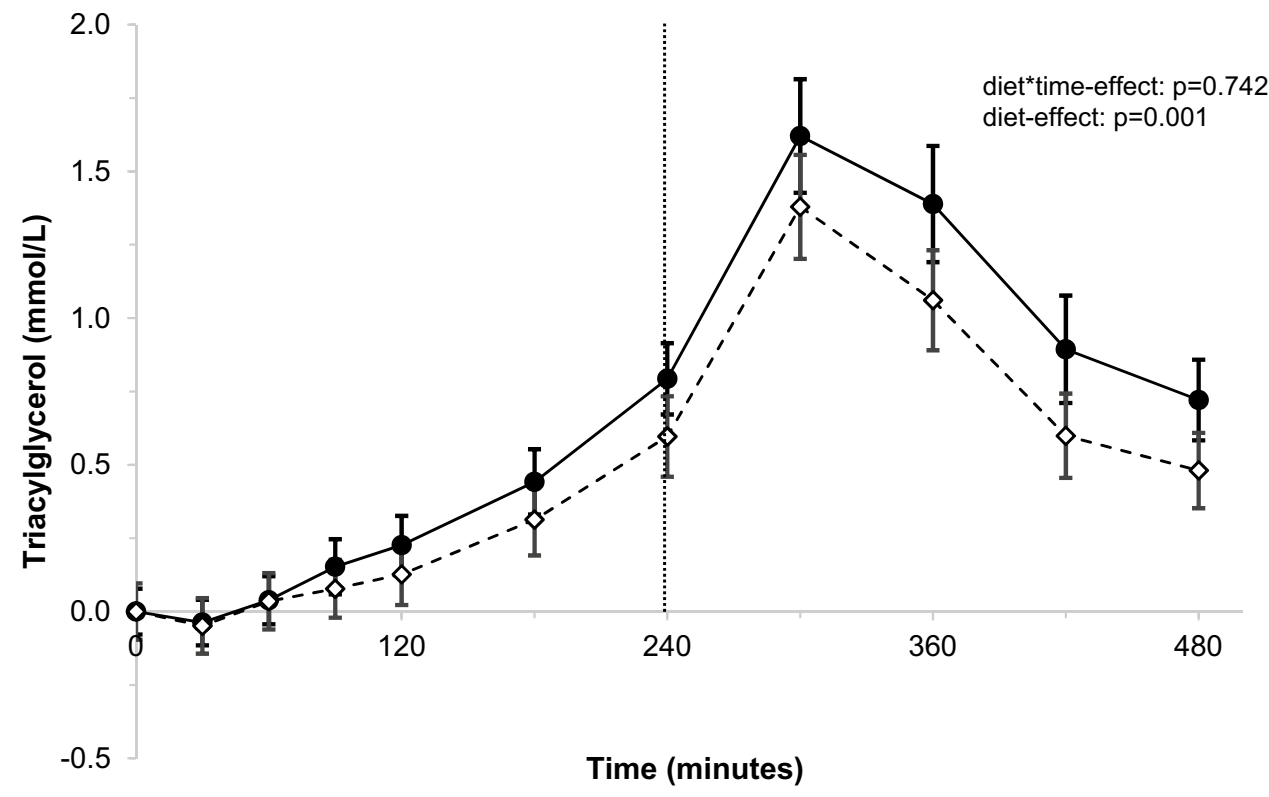


Fig. 2 Postprandial changes in apolipoprotein B48 (apoB48; $\mathrm{mg} / \mathrm{L}$ ) over time after meals rich in palmitic acid $(\bullet)$ or stearic acid $(\diamond)^{\mathrm{a}}$. ${ }^{\mathrm{a}} \mathrm{ApoB} 48$ concentrations were measured at baseline, and $30,60,90,120,180,240$, 300,360 , and $480 \mathrm{~min}$ after meal intake. After $240 \mathrm{~min}$, a second meal was consumed that was similar to the first meal. Postprandial time curves were analyzed using linear mixed models. $N=32$. A significant overall diet-effect was observed $(p=0.002)$
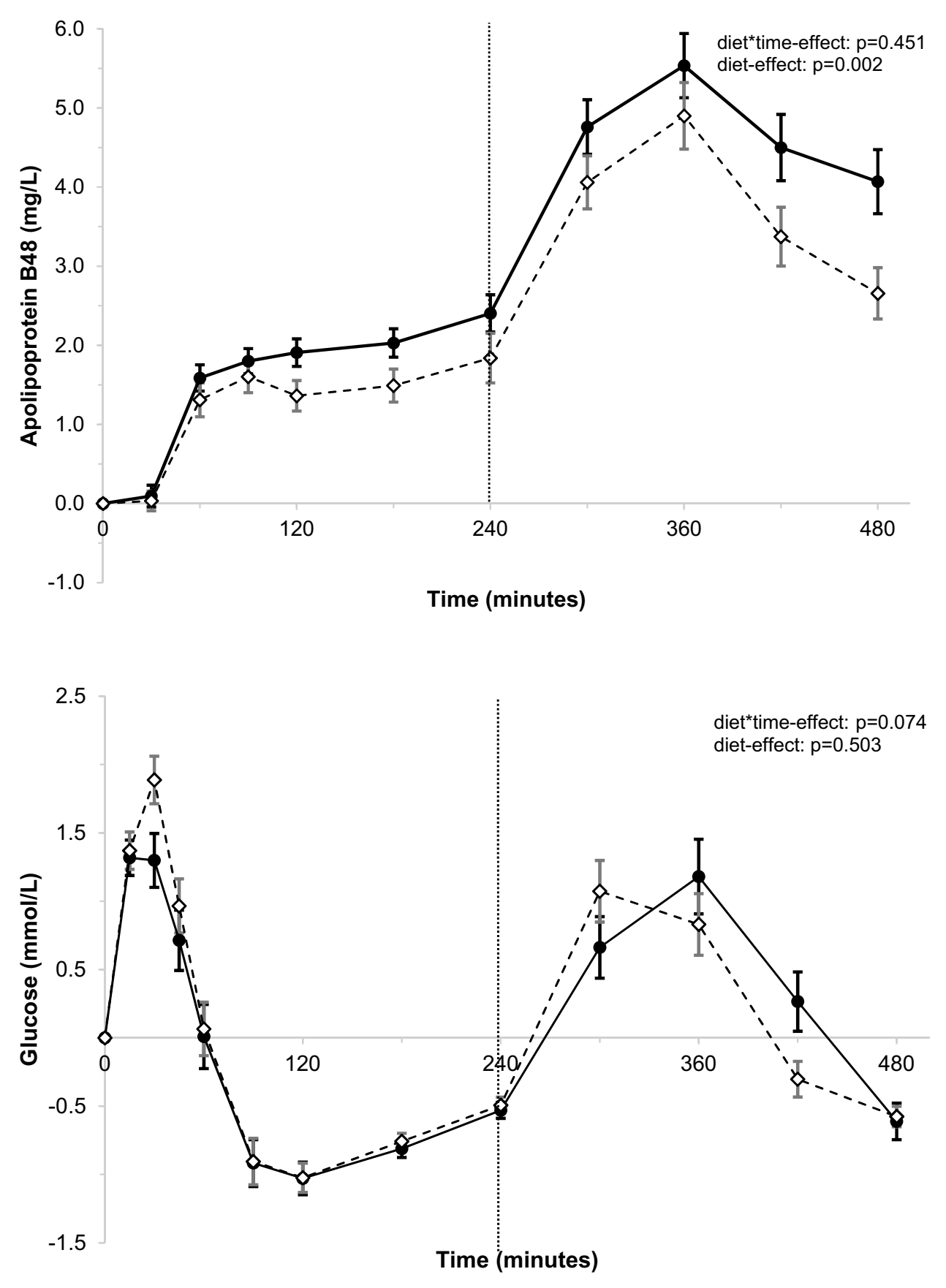

Fig. 3 Postprandial changes in glucose (mmol/L) over time after meals rich in palmitic acid $(\bullet)$ or stearic acid $(\diamond)^{\mathrm{a}}$. ${ }^{\mathrm{a}}$ Glucose concentrations were measured at baseline, and 15, 30, 45, 60, 90, 120, 180, 240, 300, 360, and $480 \mathrm{~min}$ after meal intake. After 240 min, a second meal was consumed that was similar to the first meal. Postprandial time curves were analyzed using linear mixed models. $N=32$ the $4 \mathrm{~h}$ after the first meal, the $\mathrm{iAUC}_{4-8 \mathrm{~h}}(p=0.095)$ and the Glucose $_{\max 4-8 \mathrm{~h}}(p=0.064)$ after the second meal tended to be lower after stearic-acid intake.

Differences in postprandial changes at the various timepoints in insulin concentrations were comparable between the palmitic-acid and stearic-acid meals ( $p=0.248$ for diet*time interaction; Fig. 4), and also no diet-effects were observed $(p=0.636)$. The $\mathrm{iAUC}_{0-8 \mathrm{~h}}(p=0.404)$ and Insulin $_{\max 0-8 \mathrm{~h}}(p=0.483)$ did not differ between palmiticacid and stearic-acid intakes, and similar results were observed during the $4 \mathrm{~h}$ after the first meal (Supplemental table 7). After the second meal, the insulin $\mathrm{iAUC}_{4-8 \mathrm{~h}}$ tended to be lower after intake of stearic acid $(p=0.064)$, while insulin $_{\max 4-8 \mathrm{~h}}$ was not different $(p=0.115)$.

Postprandial changes in C-peptide concentrations at the various timepoints differed between palmitic acid and stearic acid ( $p=0.020$ for diet*time interaction; Fig. 5). Compared with palmitic acid, stearic-acid intake resulted in significantly higher C-peptide concentrations 30 and $300 \mathrm{~min}$ postprandially $(+1.01 \mathrm{ng} / \mathrm{mL} ; p=0.011$ and $+1.16 \mathrm{ng} /$ $\mathrm{mL} ; p=0.004$ respectively), but in lower concentrations at $420 \mathrm{~min}(-0.96 \mathrm{ng} / \mathrm{mL} ; p=0.015)$. No differences between 
Fig. 4 Postprandial changes in insulin $(\mu \mathrm{U} / \mathrm{mL})$ over time after meals rich in palmitic acid $(\bullet)$ or stearic acid $(\diamond)^{\mathrm{a}}$. ${ }^{\mathrm{a}}$ Insulin concentrations were measured at baseline, and 15, 30, 45, 60, $90,120,180,240,300,360$, and $480 \mathrm{~min}$ after meal intake. After $240 \mathrm{~min}$, a second meal was consumed that was similar to the first meal. Postprandial time curves were analyzed using linear mixed models. $N=32$
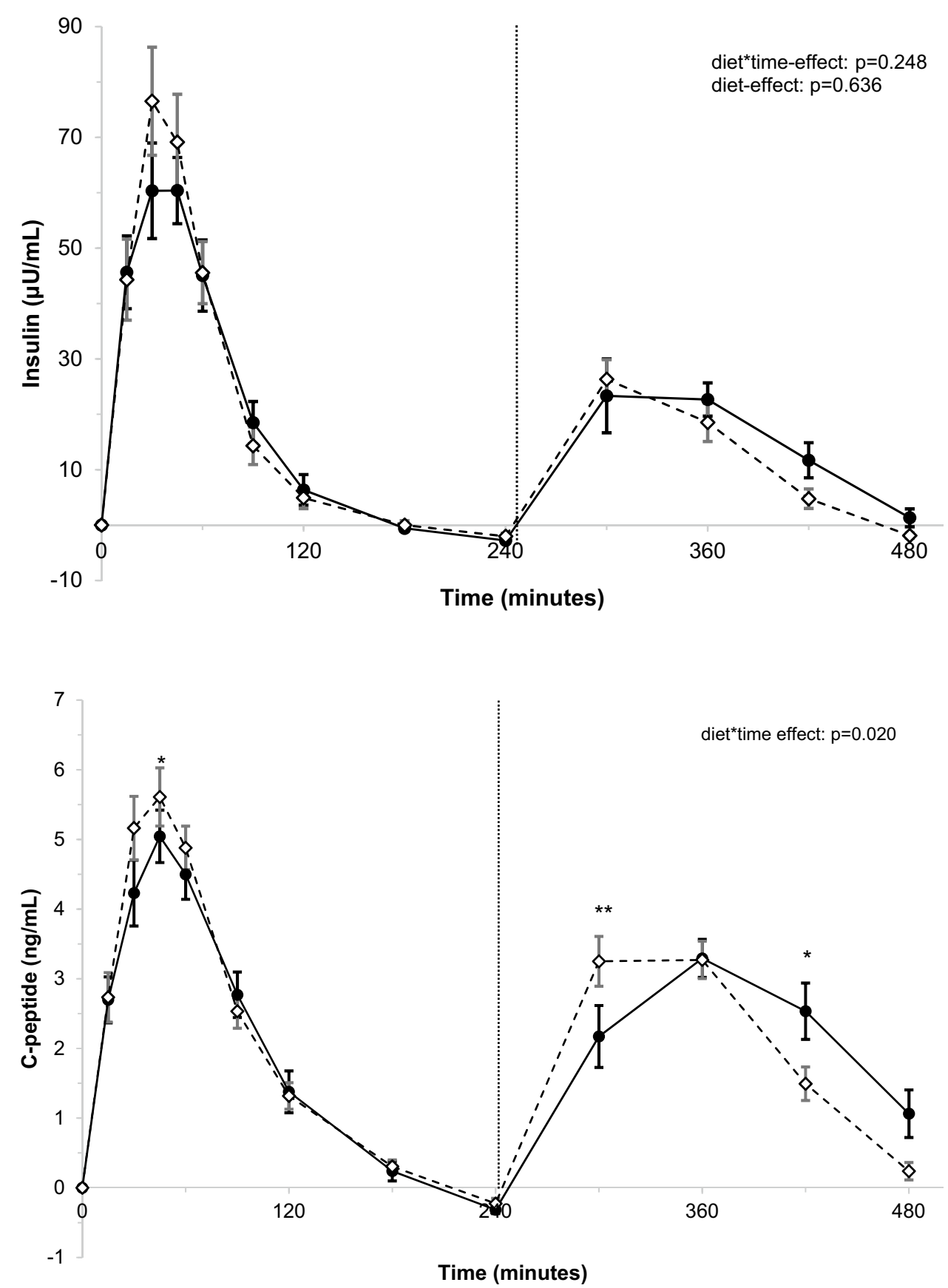

Time (minutes)
Fig. 5 Postprandial changes in C-peptide (ng/mL) over time after meals rich in palmitic acid $(\bullet)$ or stearic acid $(\diamond)^{\mathrm{a}}$. ${ }^{\mathrm{a} C} \mathrm{C}$-peptide concentrations were measured at baseline, and 15, 30, 45, $60,90,120,180,240,300,360$, and 480 min after meal intake. After 240 min, a second meal was consumed that was similar to the first meal. Postprandial time curves were analyzed using linear mixed models. $N=32$. A significant diet*time interaction was observed $(p=0.020)$ palmitic-acid and stearic-acid intakes were found in iAUCs and peak values over the total 8 -h follow up, or in those over the $4 \mathrm{~h}$ follow up after the first and second meals (Supplemental table 8).

The postprandial time curves of NEFAs were different between the palmitic-acid and stearic-acid meals ( $p<0.001$ for diet*time interaction; Fig. 6). NEFA concentrations were lower after the stearic-acid meals at T300 ( $177 \mu \mathrm{mol} / \mathrm{L} ; p<0.001)$, T360 (- $181 \mu \mathrm{mol} / \mathrm{L}$; $p<0.001)$, and T420 (-111 $\mathrm{mol} / \mathrm{L} ; p=0.001)$. The postprandial $\mathrm{dAUC}_{0-8 \mathrm{~h}}(p=0.005)$ and maximal decrease of NEFA ( NEFA $\left._{\max 0-8 \mathrm{~h} ;} p=0.026\right)$ were more pronounced after stearic-acid intake (Supplemental table 9). Similarly, in the $4 \mathrm{~h}$ after the first meals, the $\mathrm{dAUC}_{0-4 \mathrm{~h}}$ was more pronounced after intake of stearic acid $(p=0.025)$ and the maximal decreases tended to be more pronounced $\left(\mathrm{NEFA}_{\max 0-4 \mathrm{~h}} ; p=0.054\right)$. After the second meal, the $\mathrm{dAUC}_{4-8}$ also tended to be more pronounced after stearicacid intake $(p=0.054)$, but the maximal decreases did not differ $\left(\mathrm{NEFA}_{\max 4-8 \mathrm{~h}} ; p=0.499\right)$. 
Fig. 6 Postprandial changes in non-esterified fatty acids (NEFA; $\mu \mathrm{mol} / \mathrm{L}$ ) over time after meals rich in palmitic acid $(\bullet)$ or stearic acid $(\diamond)^{\mathrm{a}}$. ${ }^{\mathrm{a}} \mathrm{NEFA}$ concentrations were measured at baseline, and 15, 30, 45, 60, 90, 120, 180, 240, 300, 360, and $480 \mathrm{~min}$ after meal intake. After 240 min, a second meal was consumed that was similar to the first meal.. Postprandial time curves were analyzed using linear mixed models. $N=32$. A significant diet*time interaction was observed $(p<0.001)$

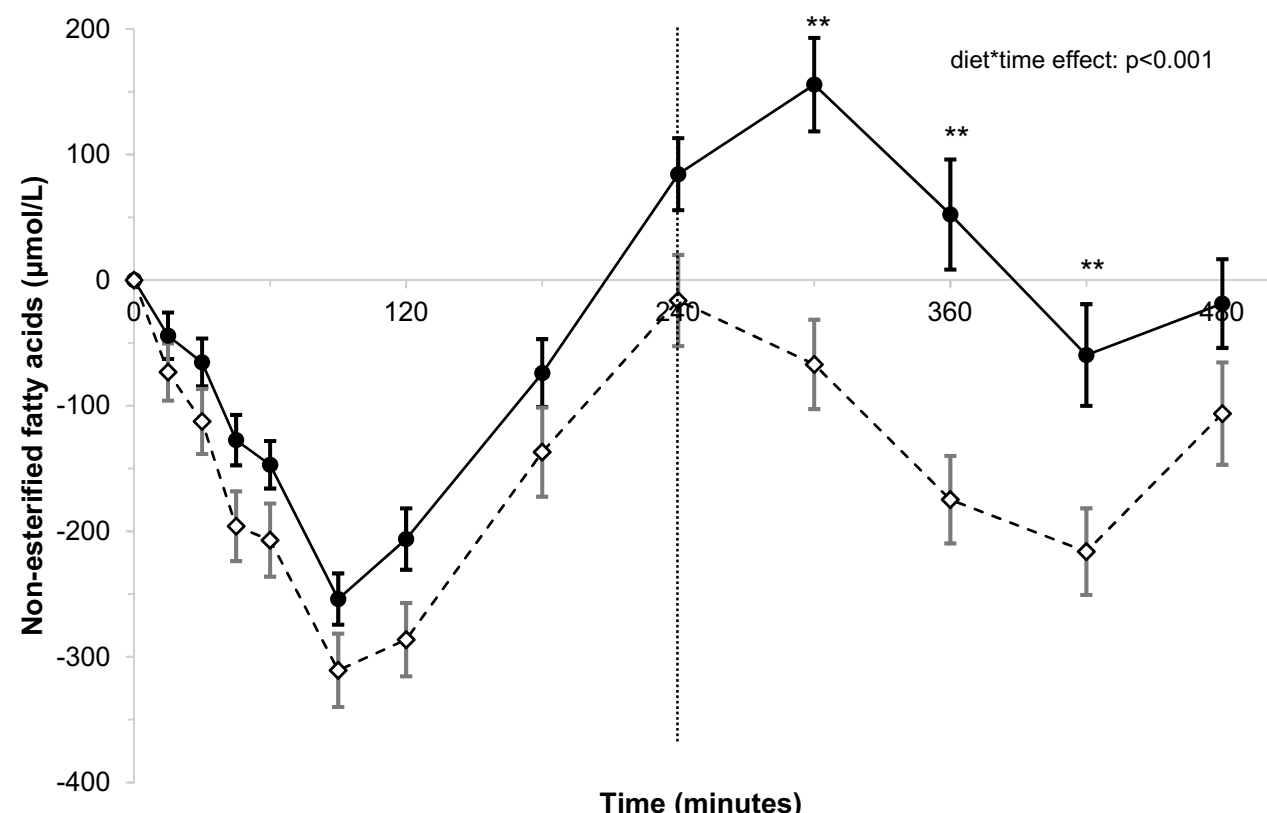

\section{Discussion}

Results of this double-blind randomized study indicate that fats rich in palmitic acid or stearic acid, the two major saturated fatty acids in most Western diets, differently affect postprandial metabolism. Effects were studied after intakes of two consecutive mixed meals high in palmitic acid- or stearic acid-rich fat blends and tests were performed after subjects had consumed for 4 weeks diets rich in the corresponding fatty acid.

\section{Postprandial lipemia}

Postprandial lipemia was decreased after intake of the stearic acid-rich meals compared with the palmitic acid-rich meals, as indicated by lower TAG and apoB48 concentrations. Differences between the meals, as indicated by the iAUCs, were in particular evident after the first meal. Earlier studies on postprandial lipid responses between palmitic acid and stearic acid-rich meals were not consistent. In most studies [7-11], no clear differences were observed, although in one study lower TAG concentrations were reported $3 \mathrm{~h}$ after intake of the meal rich in stearic acid [9]. In two other studies, however, lower TAG concentrations were observed after intake of a stearic acid-rich meal (lard) as compared with a palmitic acid-rich meal (palm olein) [5, 6]. These lower TAG concentrations - which agree with our findings-may relate to the physical characteristics of the fat sources used, especially to those of stearic acid, and not by the fatty-acid composition per se. It has been suggested that postprandial lipemia is attenuated if the fat is not fully liquid at $37^{\circ} \mathrm{C}[6$, 19]. Indeed, lard had a higher percentage of solids at $37^{\circ} \mathrm{C}$ than palm olein [6] and in our study the stearic-acid fat blend also had more solids at $37{ }^{\circ} \mathrm{C}$ than the palmitic-acid blend. We also observed a lower apoB48 response after intake of the stearic-acid meals. As each chylomicron particle carries one apoB 48 , this indicates that the number of chylomicrons after stearic-acid intake was lower. In only one other study, apoB48 responses were measured and concentrations tended to be lower after lard intake than after palm-olein intake [6]. This is in line with the hypothesis that a higher solid fat content at $37^{\circ} \mathrm{C}$ decreases or delays the absorption rate, resulting in less formation of chylomicron particles and consequently attenuated lipemia [19].

Participants consumed a second, identical meal $4 \mathrm{~h}$ after intake of the first meal. After this second meal, differences between palmitic acid and stearic acid on postprandial lipemia were less pronounced. As TAG concentrations were still increasing $4 \mathrm{~h}$ after the first meal, it can be speculated that not all the fat was absorbed within this time period, thereby increasing variability in responses and masking possible differences between the two saturated fatty acids after the second meal.

Irrespective of the fatty-acid composition of the meals, serum TAG concentrations already peaked $1 \mathrm{~h}$ after the second meal and then started to decrease, while TAG concentrations increased for up to $4 \mathrm{~h}$ after the first meal. The rapid increase in TAG after the second meal may have been caused by a release of chylomicron particles that were already formed after the first meal and stored within the enterocyte [15]. This phenomenon was also observed by Baumgartner et al. [20]. In contrast, Tushuizen et al. observed a TAG peak $2 \mathrm{~h}$ after a second meal [21]. However, in that study blood was sampled at 2 -h intervals and the mixed meals were 
provided as solid foods, while we and Baumgartner et al. [21] sampled every hour and provided the meals as a shake, which may have increased gastric emptying. Remarkably, the apoB48 peak after the second meal occurred $1 \mathrm{~h}$ later than the TAG peak, while after the first meal, both apoB48 and TAG concentrations increased continuously. This was also observed by Baumgartner et al. [20] especially in participants aged between 53 and 69 years of age, which is comparable to the age of our study population. The mechanism underlying this delayed apoB48 peak compared with the TAG peak remains to be determined, but it is possible that during the first hour after the second meal, larger TAGrich chylomicrons are secreted, as suggested by the higher TAG:apoB48 ratio at $300 \mathrm{~min}$ postprandially compared to 60 min. Alternatively, it can be speculated that the contribution of VLDL-TAG to total TAG in the circulation is larger in the first hour after the second meal.

\section{Postprandial glycemia}

C-peptide concentrations were higher after the first stearic acid-rich meal and peaked earlier after the second stearicacid rich meal as compared with corresponding palmiticacid meals. Postprandial insulin responses were however not different between palmitic-acid and stearic-acid intakes. For glucose, a comparable pattern as for C-peptide was observed, although these differences did not reach statistical significance. After both meals, postprandial NEFA suppression was more pronounced after stearic-acid intake, in particular after the second meal. Earlier studies that used a single fat-rich meal challenge did not observe any differences between palm olein or palm oil and lard on glucose [5, 13], insulin [5, 8, 13], or C-peptide [13] responses. In two studies, postprandial NEFA concentrations were lower after lard than after palm olein intake [5, 6]. Interesterified palm olein, however, had the same effect as lard, indicating that the observed differences in NEFA were most likely due to the physical characteristics of the fats rather than the fattyacid compositions [6]. Circulating NEFAs are the resultant of adipocyte lipolysis, NEFA spillover from hydrolysis of circulating TAG-rich lipoproteins, and NEFA uptake and re-esterification [22]. We can only speculate which of these processes was mostly affected by intakes of the palmitic or stearic acid-rich fat blends. Linked to the lower TAG concentrations, the larger decrease in NEFA after stearicacid intake may be caused by less spillover of NEFAs after hydrolysis of TAG-rich lipoproteins. In addition, increased or earlier insulin secretion after the stearic-acid meals as suggested by differences in C-peptide concentrations may have played a role.

In contrast to postprandial lipemia, differences between palmitic-acid and stearic-acid intakes on parameters related to postprandial glycemia were most pronounced after the second meal. This so-called second-meal effect emphasizes the importance of including second meal challenges to understand dietary effects on postprandial metabolism.

\section{Limitations and conclusions}

In the present study, blood was sampled at 15 min-intervals after the first meals and at $1 \mathrm{~h}$-intervals after the second meals. We can therefore not exclude that the true peaks of glucose, insulin, and C-peptide after the second meals were missed. Also, palmitic acid- or stearic acid-rich meal challenges were performed after 4 -week diets enriched with the corresponding fatty acid. Although this is certainly a strength as results more mimic the real life situation, it is not known to what extent our results can be compared to acute studies.

In conclusion, our data demonstrate that the fat blend rich in stearic acid lowered postprandial lipemia as compared with the fat blend rich in palmitic acid. These effects were most pronounced after intake of the first meal. Differences in C-peptide and NEFA concentrations were more evident after intake of the second meal, i.e. intake of the stearic acid-rich fat resulted in an earlier peak of C-peptide concentrations and more pronounced decrease in NEFA concentrations. Translation of these findings into health effects on the longterm needs further study.

Supplementary Information The online version contains supplementary material available at https://doi.org/10.1007/s00394-021-02530-2.

Acknowledgements We thank Maud Beckers for performing the biochemical analyses and Cara op 't Eyndt for dietary assistance.

Authors' contributions The author's responsibilities were as followsRPM, JP, and WAMB designed research; MAvR conducted research; RPM and MAvR analyzed data; RPM and MAvR wrote the first draft of the paper; RPM had primary responsibility for final content. All authors have read and approved the final manuscript.

Funding This trial was funded by Unilever R\&D Wageningen (before divesting its spreads business and since July 2, 2018 operating under the name Upfield ${ }^{\mathrm{TM}}$ ).

Availability of data and materials Not applicable.

Code availability Not applicable.

\section{Compliance with ethical standards}

Conflicts of interest MAvR, JP and RPM report no conflict of interest. WAMB and PLZ were employed by Unilever R\&D Wageningen, the Netherlands.

Ethics approval The Medical Ethical Committee of the MUMC + had approved the protocol. 
Consent to participate All participants gave their written informed consent before entering the study.

Consent for publication All participants gave their written informed consent before entering the study.

Open Access This article is licensed under a Creative Commons Attribution 4.0 International License, which permits use, sharing, adaptation, distribution and reproduction in any medium or format, as long as you give appropriate credit to the original author(s) and the source, provide a link to the Creative Commons licence, and indicate if changes were made. The images or other third party material in this article are included in the article's Creative Commons licence, unless indicated otherwise in a credit line to the material. If material is not included in the article's Creative Commons licence and your intended use is not permitted by statutory regulation or exceeds the permitted use, you will need to obtain permission directly from the copyright holder. To view a copy of this licence, visit http://creativecommons.org/licenses/by/4.0/.

\section{References}

1. Pirillo A, Norata GD, Catapano AL (2014) Postprandial lipemia as a cardiometabolic risk factor. Curr Med Res Opin 30(8):14891503. https://doi.org/10.1185/03007995.2014.909394

2. Fava S (2008) Role of postprandial hyperglycemia in cardiovascular disease. Expert Rev Cardiovasc Ther 6(6):859-872. https:// doi.org/10.1586/14779072.6.6.859

3. Cholesterol Treatment Trialists' (CTT) Collaborators (2012) The effects of lowering LDL cholesterol with statin therapy in people at low risk of vascular disease: meta-analysis of individual data from 27 randomised trials. Lancet 380(9841):581-590. https:// doi.org/10.1016/S0140-6736(12)60367-5

4. Mensink RP, Zock PL, Kester A, Katan MB (2003) Effects of dietary fatty acids and carbohydrates on the ratio of serum total to HDL cholesterol ratio and on serum lipids and apolipoproteins: a meta-analysis of 60 controlled trials. Am J Clin Nutr 77(5):11461155. https://doi.org/10.1093/ajen/77.5.1146

5. Teng KT, Nagapan G, Cheng HM, Nesaretnam K (2011) Palm olein and olive oil cause a higher increase in postprandial lipemia compared with lard but had no effect on plasma glucose, insulin and adipocytokines. Lipids 46(4):381-388. https://doi.org/10. 1007/s11745-010-3516-y

6. Sanders TA, Filippou A, Berry SE, Baumgartner S, Mensink RP (2011) Palmitic acid in the sn-2 position of triacylglycerols acutely influences postprandial lipid metabolism. Am J Clin Nutr 94(6):1433-1441. https://doi.org/10.3945/ajcn.111.017459

7. Mennen L, de Maat M, Meijer G, Zock P, Grobbee D, Kok F, Kluft C, Schouten E (1998) Factor VIIa response to a fat-rich meal does not depend on fatty acid composition: a randomized controlled trial. Arterioscler Thromb Vasc Biol 18(4):599-603

8. Jensen J, Bysted A, Dawids S, Hermansen K, Holmer G (1999) The effect of palm oil, lard, and puff-pastry margarine on postprandial lipid and hormone responses in normal-weight and obese young women. Br J Nutr 82(6):469-479

9. Sanders TA, de Grassi T, Miller GJ, Morrissey JH (2000) Influence of fatty acid chain length and cis/trans isomerization on postprandial lipemia and factor VII in healthy subjects (postprandial lipids and factor VII). Atherosclerosis 149(2):413-420
10. Tholstrup T, Sandstrom B, Bysted A, Holmer G (2001) Effect of 6 dietary fatty acids on the postprandial lipid profile, plasma fatty acids, lipoprotein lipase, and cholesterol ester transfer activities in healthy young men. Am J Clin Nutr 73(2):198-208

11. Meng H, Matthan NR, Wu D, Li L, Rodriguez-Morato J, Cohen R, Galluccio JM, Dolnikowski GG, Lichtenstein AH (2019) Comparison of diets enriched in stearic, oleic, and palmitic acids on inflammation, immune response, cardiometabolic risk factors, and fecal bile acid concentrations in mildly hypercholesterolemic postmenopausal women-randomized crossover trial. Am J Clin Nutr 110(2):305-315. https://doi.org/10.1093/ajen/nqz095

12. Berry SE, Miller GJ, Sanders TA (2007) The solid fat content of stearic acid-rich fats determines their postprandial effects. Am J Clin Nutr 85(6):1486-1494

13. Filippou A, Berry SE, Baumgartner S, Mensink RP, Sanders TA (2014) Palmitic acid in the sn-2 position decreases glucosedependent insulinotropic polypeptide secretion in healthy adults. Eur J Clin Nutr 68(5):549-554. https://doi.org/10.1038/ejcn.2014. 49

14. Evans K, Kuusela PJ, Cruz ML, Wilhelmova I, Fielding BA, Frayn KN (1998) Rapid chylomicron appearance following sequential meals: effects of second meal composition. Br J Nutr 79(5):425429. https://doi.org/10.1079/bjn19980072

15. Silva KD, Wright JW, Williams CM, Lovegrove JA (2005) Meal ingestion provokes entry of lipoproteins containing fat from the previous meal: possible metabolic implications. Eur J Nutr 44(6):377-383. https://doi.org/10.1007/s00394-004-0538-3

16. van Rooijen MA, Plat J, Blom WAM, Zock PL, Mensink RP (2020) Dietary stearic acid and palmitic acid do not differently affect ABCA1-mediated cholesterol efflux capacity in healthy men and postmenopausal women: a randomized controlled trial. Clin Nutr. https://doi.org/10.1016/j.clnu.2020.08.016

17. Dubois C, Beaumier G, Juhel C, Armand M, Portugal H, Pauli AM, Borel P, Latge C, Lairon D (1998) Effects of graded amounts (0-50 g) of dietary fat on postprandial lipemia and lipoproteins in normolipidemic adults. Am J Clin Nutr 67(1):31-38. https://doi. org/10.1093/ajen/67.1.31

18. Matthews JN, Altman DG, Campbell MJ, Royston P (1990) Analysis of serial measurements in medical research. BMJ 300(6719):230-235. https://doi.org/10.1136/bmj.300.6719.230

19. Berry SE (2009) Triacylglycerol structure and interesterification of palmitic and stearic acid-rich fats: an overview and implications for cardiovascular disease. Nutr Res Rev 22(1):3-17. https://doi. org/10.1017/s0954422409369267

20. Baumgartner S, Mensink RP, Plat J (2016) Effects of a plant sterol or stanol enriched mixed meal on postprandial lipid metabolism in healthy subjects. PLoS ONE 11(9):e0160396. https://doi.org/ 10.1371/journal.pone.0160396

21. Tushuizen ME, Nieuwland R, Scheffer PG, Sturk A, Heine RJ, Diamant M (2006) Two consecutive high-fat meals affect endothelial-dependent vasodilation, oxidative stress and cellular microparticles in healthy men. J Thromb Haemost 4(5):1003-1010. https://doi.org/10.1111/j.1538-7836.2006.01914.x

22. Jackson KG, Wolstencroft EJ, Bateman PA, Yaqoob P, Williams CM (2005) Acute effects of meal fatty acids on postprandial NEFA, glucose and apo E response: implications for insulin sensitivity and lipoprotein regulation? Br J Nutr 93(5):693-700. https:// doi.org/10.1079/bjn20051410 